\title{
A Case of Contrast Enhanced Cystic Mass of External Ear Canal Diagnosed as Engorged Hard Tick
}

\author{
Min Young Lee and Jae Yun Jung \\ Department of Otorhinolaryngology-Head \& Neck Surgery, Dankook University Hospital, Cheonan, Korea
}

Received December 28, 2017

Revised February 6, 2018

Accepted February 27, 2018
A 58-year-old female complained earfullness and otalgia and came to the clinic. Dark gray colored cystic mass obstructing the left ear canal was observed during the endoscopic exam. Contrast enhanced CT image showed an oval shape soft tissue lesion in the mid part of the external auditory canal with a homogenous increased contrast uptake. The mass was removed under microscopic view at outpatient clinic. While dissecting the mass in the ear canal, a pod like structure was visualized. The external organism was densely adherent to the skin and turned out to be an engorged hard tick. Considering the final diagnosis, contrast uptake observed in the CT scan implicates the patient communication of blood between host and parasite. This unique image finding differs from a benign mass lesion in the ear canal was one of the differential diagnosis points for the lesion. This article is reporting the case of tick bite in the ear canal with a rare CT scan finding.

J Audiol Otol 2018;22(3):167-169

\section{Introduction}

Foreign body on the external ear canal is not a rare finding, children often visit the ER after inserting a small toy into their ear canal and adults often come with cotton wool or broken matchsticks [1]. In the rural area, small organism such as insects [2], mites, and ticks [3] can be annoying due to their sound and bite pain. Mostly, these foreign bodies are easily diagnosed with an endoscopic exam of the tympanic membrane and external ear canal which rarely obstruct the ear canal.

Fatal diseases such as Lyme disease and severe fever with thrombocytopenia syndrome can occur following a tick bite $[4,5]$. Elimination of the parasite is necessary to halt the progression into a fatal disease. Tick bites were mostly observed in the head, neck, and extremities. Tick bites occurring in the ear canal is extremely rare; only one case was reported in Korea [6]. Furthermore, when the tick is engorged, it could be difficult to diagnose since the whole morphology of the parasite is not identified. Blood communication between host and

This is an Open Access article distributed under the terms of the Creative Commons Attribution Non-Commercial License (http://creativecommons.org/licenses/by-nc/4.0/) which permits unrestricted non-commercial use, distribution, and reproduction in any medium, provided the original work is properly cited. parasite is expected, but not much evidence of spreading contrast agent from the host to parasite exists.

We are reporting a successful removal of a tick presenting as a cystic mass like lesion obstructing the ear canal, inducing pain and hearing loss without complication. In addition, we are providing the unique feature of this external organism observed in enhanced CT scan.

\section{Case Report}

A 58-year-old female patient who lives in the rural mountain came to the clinic complaining of hearing loss and otalgia in her left ear which started one week prior to the visit. She did not have any past medical history except for a previous thyroidectomy operation. The left ear canal was impacted with a dark gray colored cystic lesion (Fig. 1A). The cystic mass surrounded by small crusts was observed in the distal part of external ear canal skin. A pod like structure was visualized at the posterior superior quadrant of the cystic lesion (Fig. 1B) and the external organism was densely adherent to the skin medial to the pod (Supplementary Video 1 in the online-only Data Supplement).

At first, the patient was examined by a resident to exclude 

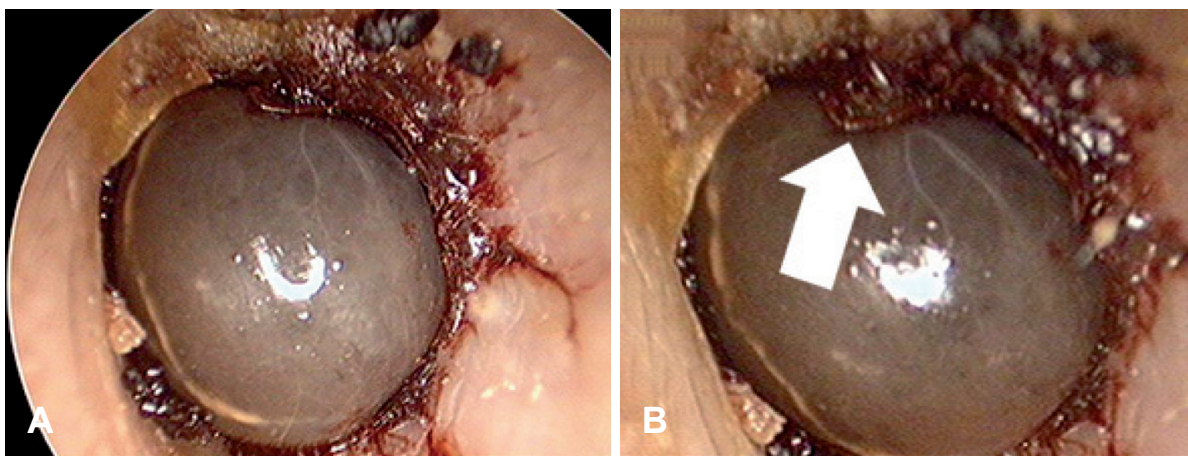

Fig. 1. Endoscopic image of the cystic ear canal lesion. A: At the initial endoscopic view of the cystic ear canal lesion, a dark gray color with cystic nature was observed. B: With a closer observation, at the posterior and superior part of the cystic lesion, a pod like structure was visible (arrow).

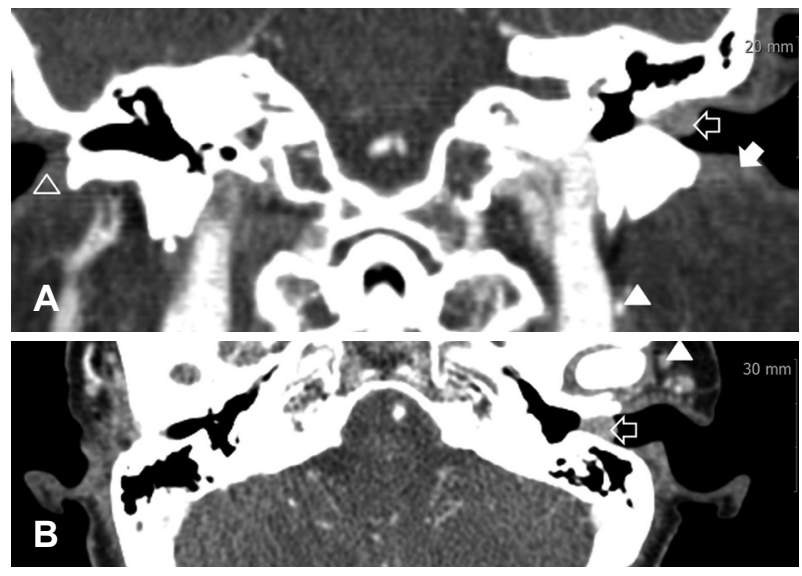

Fig. 2. Images of the enhanced temporal bone CT scan showing contrast enhancement. Coronal (A) and axial (B) enhanced CT image of the cystic lesion showed a heterogeneous and moderate enhancement in the ear canal (empty arrow). HU (Hounsfield unit) of cystic lesion (empty arrow) was between 100-150. In contrast, $\mathrm{HU}$ of the ipsilesional (arrow) and contralateral (empty arrow head) ear cartilage was below 100. For reference, vein (arrow head) showed a HU of 130, air with -922 and bone with 1000 .

the other cystic lesion on the external ear canal and an enhanced CT scan was performed to evaluate the middle ear. The enhanced CT image showed a slightly enhanced oval shaped soft tissue lesion in the mid part of the external auditory canal (Fig. 2). The lesion appeared heterogeneous and moderately enhanced in the ear canal (empty arrow). HU (Hounsfield unit) of the cystic lesion (empty arrow) was between 100-150. In contrast, HU of ipsilesional (arrow) and contralateral (empty arrow head) ear cartilage was below 100. For reference, a vein (arrow head) showed a HU of 130. Contrast enhancement similar to vein was observed and could be a differential point of the lesion. This lesion did not invade the middle ear and mastoid cavity with the ossicular chain intact. The patient came to an ENT specialist after the CT scan, and the cystic lesion appears like an organic structure. The cystic lesion was gently dissected from the external ear canal skin with ear forceps and sharp instrument. However, en bloc removal of the cystic lesion was not easy due to its dense adhesion to the skin of ear canal. Thus, after rupturing the cystic part, the cys-

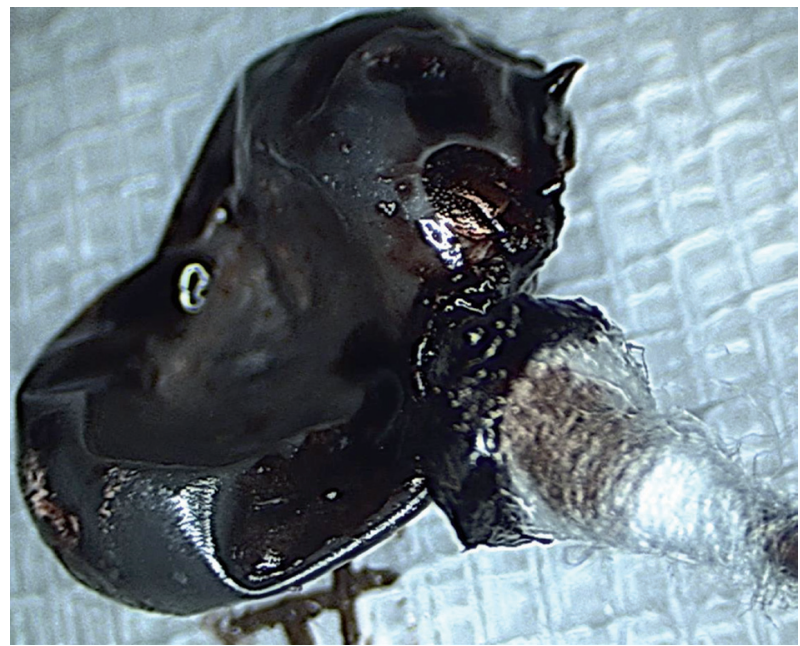

Fig. 3. Mass removed from the external ear canal. The external organism was removed from the ear canal showing the pods and cystic body.

tic lesion was removed with grasping forceps (Fig. 3). The extracted specimen was suspected to be a haematophagic organism blocking the external ear canal. After the removal, symptom of the patient was relieved and did not have any systemic symptoms such as fever or myalgia.

\section{Discussion}

In the present case, the patient had a symptom of hearing loss and ear pain and removing the pathogenic organism relieved the symptom immediately. However, there are more things to consider when treating a mite or tick bite in the clinic. An interesting finding was that contrast enhancement of external organism which suggests that blood transfer from the matrix is not minimal. Therefore, any secondary symptom of disease, which could be transferred by these organisms, should be assessed in detail because there are several secondary diseases caused by these organisms. There are possibilities of systemic diseases such as Rocky Mountain fever [7], tularemia [8], Lyme disease [9], and Crimean-Congo hemorrhag- 
ic fever [10]. These diseases should not be underestimated due to relatively higher incidence in specific regions, morbidity and even mortality.

This case is the first case to demonstrate a CT scan image showing the contrast enhancement of an organism in the ear canal. The engorged tick should be distinguished from an epidermoid cyst. Epidermoid cysts have soft and tender surface, broader base, and low dome shape, while the engorged tick in this case had a firm surface, narrower base of contact, and taller dome shape. Additional imaging study might help the differential diagnosis, since non-enhancement of a cystic fluid lesion is expected in an epidermoid cyst compared to the enhancement of the total cystic mass from this case. We believe that the role of enhanced CT in diagnosis of tick bite is limited in adult cases. But in cases of children, who does not easily obey doctors or when the detailed observation of the lesion is not possible, conducting a CT scan may be beneficial for a differential diagnosis. Other benign conditions could be observed for several days but the tick should be removed immediately considering the fatal complications. Seasonal incidence of tick is high from spring to late summer, and is frequently observed in subjects who has been exposed to plants such as campers and farmers [3].

Incidence of tick bite in the ear canal is rare in humans and very rare in developed countries, with only few cases being reported $[4,6,11]$. However, reports of insects such as ticks in the auditory canal are not rare when we widen the field towards developing countries. And it is not difficult to find a cystic lesion in the ear canal such as an epidermoid cyst. Therefore distinguishing a ticks from other benign conditions is important and is one of the reasons that this case has to be published, because we can encounter such a case of tick bite and have the organism removed but consequently find out later that it had caused a fatal complication. The purpose of this article is to provide a pre-experience of this case of tick bite before an actual experience at the clinic by other clinicians as well as the public, since the complications of this tick bite such as tick-borne encephalitis [12-14] can be fatal.

\section{Supplementary Materials}

The online-only Data Supplement is available with this article at https://doi.org/10.7874/jao.2017.00388.

\section{Acknowledgments}

We thank Nathaniel Carpena for his through proof reading of this article.

\section{Conflicts of interest}

The authors have no financial conflicts of interest.

\section{REFERENCES}

1) Al-Juboori AN. Aural foreign bodies: descriptive study of 224 patients in Al-fallujah general hospital, Iraq. Int J Otolaryngol 2013; 2013:401289.

2) Kroukamp G, Londt JG. Ear-invading arthropods: a South African survey. S Afr Med J 2006;96:290-2.

3) Gökdoğan O, Çakabay T, Baran H, Karabulut B, Tasdemir C, Vatansever Z. Otoacariasis: demographic and clinical outcomes of patients with ticks in the ear canal. Braz J Otorhinolaryngol 2016;82:416-21.

4) Iwasaki S, Takebayashi S, Watanabe T. Tick bites in the external auditory canal. Auris Nasus Larynx 2007;34:375-7.

5) Prakairungthong S, Limviriyakul S, Chongvisal S. Temporal bone histopathology case of the month: acute unilateral facial paralysis in child from intra-aural hard tick. Otol Neurotol 2012;33:e81-2.

6) Sung WJ, Kim YH. A case of engorged female hard tick in the external auditory canal of an infant. Korean J Parasitol 2017;55:565-8.

7) Dantas-Torres F. Rocky Mountain spotted fever. Lancet Infect Dis 2007;7:724-32.

8) Foley JE, Nieto NC. Tularemia. Vet Microbiol 2010;140:332-8.

9) Bratton RL, Corey R. Tick-borne disease. Am Fam Physician 2005; 71:2323-30.

10) Bente DA, Forrester NL, Watts DM, McAuley AJ, Whitehouse CA, Bray M. Crimean-Congo hemorrhagic fever: history, epidemiology, pathogenesis, clinical syndrome and genetic diversity. Antiviral Res 2013;100:159-89.

11) Grady CA, McDonald DR, Poppen CF, Pritt BS. Dermacentor tick attached to tympanic membrane. Lancet 2011;378:347.

12) Alkadhi H, Kollias SS. MRI in tick-borne encephalitis. Neuroradiology 2000;42:753-5.

13) Marjelund S, Tikkakoski T, Tuisku S, Räisänen S. Magnetic resonance imaging findings and outcome in severe tick-borne encephalitis. Report of four cases and review of the literature. Acta Radiol 2004; 45:88-94.

14) Pichler A, Sellner J, Harutyunyan G, Sonnleitner A, Klobassa DS, Archelos-Garcia JJ, et al. Magnetic resonance imaging and clinical findings in adults with tick-borne encephalitis. J Neurol Sci 2017;375: 266-9.

\section{Supplementary Movie Legend}

Video 1. Endoscopic evaluation. 